P. Brekke, B. Fleck, and J. B. Gurman eds.

\title{
Magnetic Pumping at the Base of the Solar Convection Zone
}

\author{
S. M. Tobias
}

Dept. of Applied Mathematics, University of Leeds, Leeds, LS2 9JT, UK

N. H. Brummell and J. Toomre

JILA, University of Colorado, Boulder, Colorado, CO 80309-0440, USA

\begin{abstract}
The transport of magnetic flux from the convection zone into the stably-stratified tachocline is a key component of the proposed interface scenario for the solar dynamo. In this paper we give a summary of the results from three-dimensional numerical experiments (detailed in Tobias et al. 2000) of pumping of magnetic flux by turbulent, penetrative convection. We also list how the efficiency of pumping depends on the parameters of the convection.
\end{abstract}

\section{Introduction}

It is now widely believed that the 11-year solar magnetic cycle is generated by the action of a hydromagnetic dynamo (see e.g. Weiss 1994). A current paradigm under consideration for solar dynamo action is that the toroidal magnetic field is generated by the strong radial shear at the base of the solar convection zone (the tachocline) and that the weaker poloidal field is generated by the action of cyclonic eddies throughout or at the base of the convection zone. This 'interface dynamo' was first successfully modeled by Parker (1993) who stressed that the key to this dynamo work is the efficient transport of (poloidal) magnetic flux from the solar convection zone into the stably stratified tachocline and the subsequent storage of strong toroidal flux in the overshoot layer.

The transport of magnetic flux has been studied in some detail in the past. Drobyshevski \& Yuferev (1974) were the first to introduce the concept of topological pumping and argued that an asymmetry in the form of the convection could lead to a net preferential downwards transport of magnetic flux. Further kinematic studies (e.g. Arter et al. 1982) demonstrated that the pumping of magnetic flux continued to operate at higher magnetic Reynolds numbers $\left(R_{m}\right)$. In addition, they noted that extensive local stretching of the field yielded a significant increase in magnetic energy. The transport of magnetic energy in fully three-dimensional dynamo simulations has also been investigated by Nordlund et al (1992) and Brandenburg et al.(1996). In this paper we give a brief summary of the effectiveness of turbulent, compressible, penetrative convection in transporting flux from a convecting layer into a stable layer below and its implications for the solar interface dynamo scenario. Details of the setup and results of the calculations can be found in Tobias et al. (1998) and Tobias et al. (2000). 

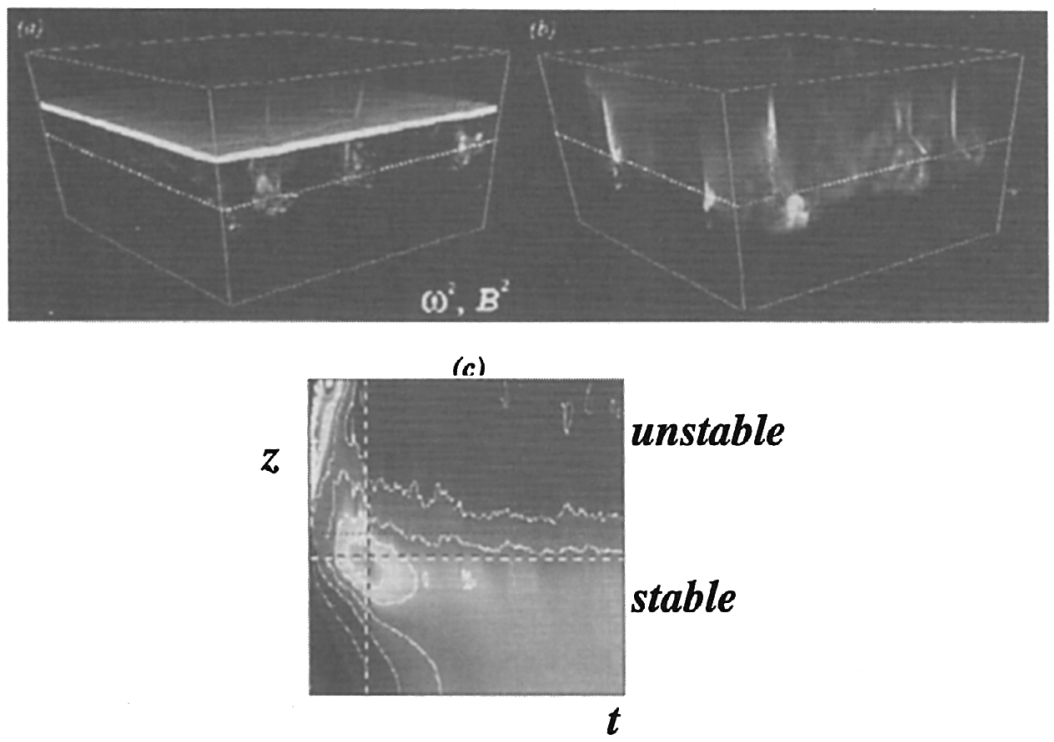

Figure 1. (a,b) Volume Renderings of magnetic energy and enstrophy. Magnetic energy ranges from bright yellow for strong fields to greens and blue tones for weak; strong enstrophy appears white-purple and is concentrated in downwards sinking plumes. (a) The initial state (b) The final state. (c) Colour-coded space-time plot of the horizontally averaged magnetic field with bright colours depicting strong average magnetic field.

\section{The model and the pumping mechanism}

We consider a three-dimensional local Cartesian domain containing a fully compressible but ideal gas confined between two horizontal, impenetrable, stressfree boundaries. We investigate penetrative convection by imposing a twolayer piecewise-polytropic stratification (in the absence of convection) within the Cartesian domain such that the upper layer is convectively unstable and the lower layer is stable. We solve the equations for the conservation of mass, momentum and energy, the equation of state for a perfect gas and the induction equation for magnetic fields as an initial value problem by a semi-implicit, hybrid finite-difference/pseudospectral scheme.

We impose a magnetic layer in the convection zone (unstable layer) of an existing hydrodynamic solution which has relaxed to a statistically-steady state. Figure $(1 \mathrm{a}, \mathrm{b})$ shows volume renderings of the magnetic fields and the enstrophy as the calculation progresses. Fields are assigned a colour and opacity according to their strength (see figure caption for details). The figure follows the calculation from the initial state of the thin horizontal layer superimposed on the penetrative convection (figure 1a). This magnetic field is then advected and stretched by both the coherent downflows and the weaker upflows. The field is pumped most efficiently by the downflows and begins to be distributed throughout and below the convective domain. As noted in Tobias et al. $(1998,2000)$ 
the strongest field is eventually concentrated at the base of the coherent downflows below the interface in the stable region. This is demonstrated in Figure 1 (c), which shows a space-time plot of the horizontally-averaged magnetic field, where the strongest field, after an initial rise, peaks just below the interface and is then redistributed in the lower stable region. For these parameter values $75-80 \%$ of the magnetic flux is pumped into stable zone on a convective timescale, demonstrating that the asymmetric compressible convection is very efficient at removing flux from the convection zone.

\section{Variation With Parameters}

In this section we list the dependence of the pumping mechanism on the parameters of the convection:

(a) The pumping mechanism is remarkably insensitive to the strength of the imposed magnetic field.

(b) Rotation decreases penetration and therefore pumping.

(c) Decreasing the supercriticality decreases the pumping.

(d) The relative stability of the two layers dramatically affects the efficiency of the pumping, with more stable lower layers leading to a decrease in magnetic pumping.

These changes in the pumping properties of the flow are due to the corresponding changes in penetration of the plumes into the stable layer.

\section{Conclusions}

The pumping mechanism that is necessary for solar interface dynamos to work efficiently arises naturally due to the action of turbulent penetrative compressible convection. Magnetic flux is efficiently transported from the convection zone into the stable layer below.

\section{References}

Arter, W., Proctor, M.R.E. \& Galloway, D.J. 1982, MNRAS, 201, 57P

Brandenburg,A., Jennings,R.L., Nordlund, A., Rieutord, M., Stein, R.F. \& Tuominen, I. 1996, J. Fluid Mech., 306, 325

Brummell, N.H., Clune T.L. \& Toomre, J. 2000, preprint

Drobyshevski, E.M., \& Yuferev, V.S. 1974, J. Fluid Mech., 65, 38

Nordlund, A., Brandenburg, A., Jennings, R.L., Rieutord, M., Ruokalainen, J., Stein, R.F., \& Tuominen, I. 1992 ApJ, 392, 647

Parker, E.N., 1993 ApJ, 408, 707

Tobias S.M., 1996 A\&A, 322, 1007

Tobias, S.M., Brummell, N.H., Clune, T.L., \& Toomre, J. 1998, ApJ, L177

Tobias, S.M., Brummell, N.H., Clune, T.L., \& Toomre, J. 2000, ApJ, submitted. 